\title{
Unsteady Couette Flow Past between Two Horizontal Riga Plates with Hall and Ion Slip Effects
}

\author{
S. Nasrin ${ }^{1, *}$, R. N. Mondal ${ }^{1}$, M. M. Alam ${ }^{2}$ \\ ${ }^{1}$ Department of Mathematics, Jagannath University, Dhaka-1100, Bangladesh \\ ${ }^{2}$ Mathematics Discipline, Khulna University, Khulna-9208, Bangladesh
}

Received November 11, 2020; Revised December 16, 2020; Accepted January 20, 2021

\section{Cite This Paper in the following Citation Styles}

(a): [1] S. Nasrin, R. N. Mondal, M. M. Alam, "Unsteady Couette Flow Past between Two Horizontal Riga Plates with Hall and Ion Slip Effects," Mathematics and Statistics, Vol. 9, No. 4, pp. 552 - 565, 2021. DOI: 10.13189/ms.2021.090414.

(b): S. Nasrin, R. N. Mondal, M. M. Alam (2021). Unsteady Couette Flow Past between Two Horizontal Riga Plates with Hall and Ion Slip Effects. Mathematics and Statistics, 9(4), 552 - 565. DOI: 10.13189/ms.2021.090414.

Copyright $\bigcirc 2021$ by authors, all rights reserved. Authors agree that this article remains permanently open access under the terms of the Creative Commons Attribution License 4.0 International License

\begin{abstract}
Riga plate is the span wise array of electrodes and permanent magnets that creates a plane surface and produced the electromagnetic hydrodynamic fluid behavior and mostly used in industrial processes with fluid flow affairs. In cases where an external application of a magnetic or electric field is required, better flow is obtained by the involvement of the Riga plate. Riga plate acts as an agent to reduce the skin friction and enhance the heat transfer phenomena. It also diminishes the turbulent effects, so that it is possible to get an efficient flow control and it increases the performance of the machine. So the numerical investigation of the unsteady Couette flow with Hall and ion-slip current effects past between two Riga plates has been studied and the numerical solutions are acquired by using explicit finite difference method and estimated results have been gained for several values of the dimensionless parameter such as pressure gradient parameter, Hall and Ion-slip parameters, modified Hartmann number, Prandtl number, and Eckert number. In this article, the importance of the modified Hartmann number on the flow profiles is immense owing to the Riga plate. The expression of skin friction and Nusselt number has been computed and the outcomes of the relevant parameters on various distributions have been sketched and presented as well as graphically.
\end{abstract}

Keywords MHD, Riga Plate, Couette Flow, Hall Current and Ion-Slip Effects, Hartmann Number, Explicit Finite Difference

\section{Introduction}

The highly conducting fluids are controlled by magneto-hydrodynamics flow with the influence of an external magnetic field known as classical MHD flow control. When the fluid is too weak in electrically conducting so that Lorentz force decreases exponentially then an external electric field must be applied to achieve an efficient flow control, this is called Electro-magneto hydrodynamic (EMHD) flow. Because of the importance of magnetic fields, the Riga plate holds a key place of its tremendous applications in the field of magneto-aerodynamics, civil engineering, mechanical engineering, chemical engineering, dust or fumes in a gas, in biomechanics and rectification of groundwater, and oil. The formulation of the Riga plate is the combination of electrodes and permanent magnets create a plane surface instead of polarity and magnetization that produce a wall parallel Lorentz force to control the fluid flow that was first introduced by Gailitis and Leilausis [1]. This order minimizes the friction and pressure drag of submarines. It is also separation of the boundary layer as well as diminishing the turbulence effects and so that a better flow pattern is obtained. When the flow passes, the Lorentz force is created by gathering the electrodes and permanent magnets in the flat surface. A turbulent channel event with a small Reynolds number is utilized the Lorentz force discovered by Berger et al. [2]. Pantokratoras and Magyari [3] proposed the electro-magneto hydrodynamic free convection fluid flow with a poor conductivity along a Riga plate. Wahidunnisa 
et al. [4] studied the heat source of a nanofluid flow through a Riga plate with viscous dissipation. Iqbal et al. [5] explored their idea on an electrically conducted Riga plate with viscous dissipation and thermal radiation of nanofluid with melting heat and they use the Keller Box scheme to obtain the solution. Ayub et al. [6] examined the EMHD nanofluid flow along an electromagnetic actuator or Riga plate. Ahmed et al. [7] carried out the mixed convection of a nanofluid flow along a vertical Riga plate with the effect of a strong suction. The physical problems of magneto-hydrodynamic flows with Hall and Ion-slip current have practical applications as electromagnetic flow meters, electromagnetic pumps and MHD power generator, aerodynamic heating, electrostatic precipitation, geophysics, astrophysics, and many engineering and industrial processes [8]. Javeri [9] investigated the combined effect of Hall and Ion slip currents, Joule heating, and viscous dissipation on the laminar MHD channel. Eraslan [10] expressed a distribution of temperature for the MHD channels with the Hall effect. The MHD laminar flow along a porous medium has significant applications in engineering and agricultural process, groundwater flows, petroleum industry, and oil and gas purification. A lot of research work has been held on the MHD steady or unsteady flows over a vertical porous plate with Hall and ion-slip under different physical effects has been studied of their wide applications. From the point of view, the effect of Hall current and ion-slip with heat and mass convection flows of an electrically conducting fluid has been discussed by several authors such as Seddeek and Aboeldahab[11] examined an unsteady free convection fluid flow with Hall currents effect of gray gas through an infinite porous plate where a strong transverse magnetic field is imputed perpendicularly to the plate. Debnath et al. [12] have studied the effects of Hall current on unsteady hydro magnetic flow past a porous plate in a rotating fluid system. Nimr and Masoud [13] carried out an unsteady free convection flow in a porous media along a flat plate. Krishna et al. [14] have carried out an unsteady free convective magneto-hydrodynamic flow with Hall and ion slip current effects through an accelerated inclined plate with rotation which is surrounded by a porous medium with the effect of inclined angle also with the change of reference frame. They have used Laplace transform to solve these problems analytically. Angirasa and Peterson [15] presented a numerical study on heat transfer in natural transmission from an isothermal vertical surface which is a stable layered to a fluid-saturated thermally stratified porous medium. Kumar and Singh [16] analyzed the heat transfer from a vertically isothermal surface with the impact of thermal stratification in a porous medium. The influence of MHD Couette flows with Hall and Ion slip current has a great importance of experimental and theoretical applications in magnetic material processing, astrophysics, polymer technology, heating electrostatics, nuclear engineering, pumps and power generators, geophysical and industrial fields. From this point of view, an unsteady Couette flow of an incompressible fluid with Ion-slip effect and with the influence of an external uniform magnetic field which is perpendicular to the plates is discussed in [17] and the same flow with uniform suction and injection past between two parallel porous plates with heat transfer is discussed in [18] by Attia. Kumar et al. [19] examined the Couette flow in three-dimensional with heat transfer through a porous medium bounded by infinite vertical porous plates. From the great interest in unsteady laminar Couette flow of its great applications in environmental, industrial, biomedical, engineering, nuclear reactors, and oil purifications, many researchers $[20,21,22]$ have expressed their views on its. Limthanakul and Pochai $[23,25]$ have given an idea about groundwater flows. They discussed a two dimensional model of polluted groundwater measured around a land fill. Koroleva [24] studied a flow of Stokes-Brinkman system through an ensemble of porous cylindrical particles with different viscosity by the cell method.

Drawing motivation from the above studies, the study aims to investigate the simultaneous effects of the unsteady Couette flow past between two horizontal Riga plates with Hall and ion slip current with electromagnetic field. The explicit finite difference method has been used as a main tool to solve the problem. Also, MATLAB R2015a has been used to calculate the results. The obtained results of different parameters have been shown graphically.

\section{Mathematical Formulation}

An incompressible laminar flow of viscous fluid between two horizontal parallel Riga plates has been considered, where one of which is moving and the other is at rest. Let the lower plate be rest at $\tilde{y}=0$ and the upper plate is moving at a distance $\tilde{y}=d$ with a velocity $\frac{\pi v}{l}$. Let the direction of the flow be taken along the $\tilde{x}$-axis, $\tilde{y}$-axis is perpendicular to the flow and the plates are parallel to the $\tilde{X} \tilde{Z}$ - plane. As a generalized Couette flow, the applied pressure gradient $\frac{\partial \tilde{p}}{\partial \tilde{x}}(=\mathrm{P})$ on the fluid is constant. Consider the plate is long enough in $X$ - direction and the fluid motion is unsteady so that the fluid may be treated as two dimensional $\left(\right.$ i.e. $\left.\frac{\partial}{\partial \tilde{z}}=0\right)$. Therefore the continuity equation leads to $\tilde{u}=\tilde{u}(\tilde{y})$. Plates are fixed at two constant temperatures $T_{1}$ for the lower plate and $T_{2}$ for the upper plate, where $T_{2}>T_{1}$. The initial temperature of the fluid is assumed to be equal to the temperature of the lower plate $T_{1}$. Due to the effect of Hall and Ion-slip current, the generalized Ohm's law may state as follows: 


$$
\mathbf{J}=\sigma(\mathbf{q} \wedge \mathbf{B})-\frac{\beta_{e}}{B_{0}}(\mathbf{J} \wedge \mathbf{B})+\frac{\omega_{e} \tau_{e} \beta_{i}}{B_{0}^{2}}(\mathbf{J} \wedge \mathbf{B}) \wedge \mathbf{B}
$$

Where, $\omega_{e} \tau_{e}=\beta_{e}$ may treat as Hall parameter. The physical model is shown in Fig.1.
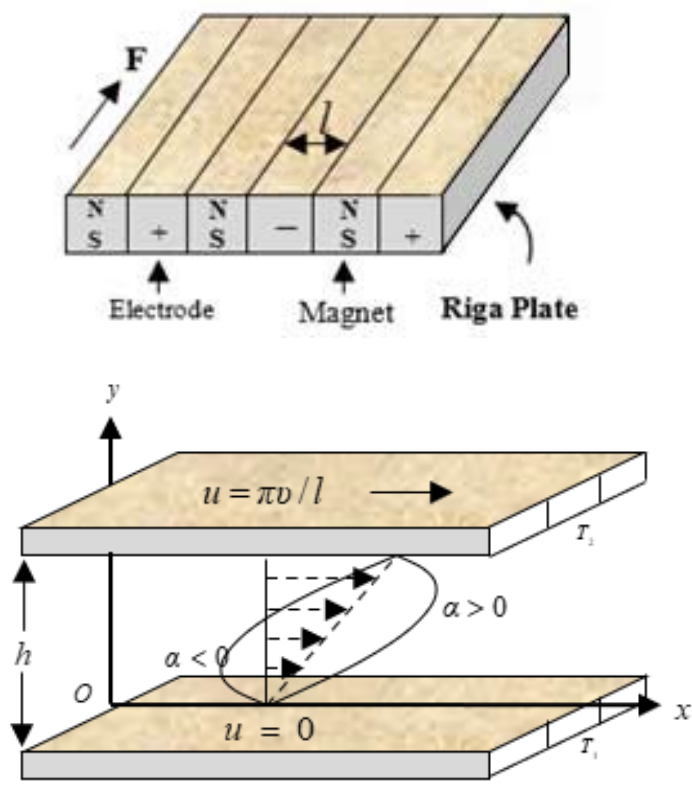

Figure 1. Physical model of the system

A uniform magnetic force is generated by the Riga plate. The Lorentz force $\bar{f}=\mathbf{J} \wedge \mathbf{B} \approx \sigma(\mathbf{E} \wedge \mathbf{B})$ is defined as magnetic forces. According to the Grinberg hypothesis this magnetic forces are defined as follows:

$$
\bar{f}=\mathbf{J} \wedge \mathbf{B}=\left(\frac{\pi}{8} J_{0} M_{0} e^{-\frac{\pi}{l} y}, 0, \frac{\pi}{8} J_{0} M_{0} e^{-\frac{\pi}{l} y}\right)
$$

Under the above assumptions of Couette flow, and Bousniques approximations, it is found the dimensional forms of the momentum and energy equations are:

$$
\begin{gathered}
\frac{\partial \tilde{u}}{\partial \tilde{t}}-v_{0} \frac{\partial \tilde{u}}{\partial \tilde{y}}=-\frac{\mathrm{P}}{\rho}+v \frac{\partial^{2} \tilde{u}}{\partial \tilde{y}^{2}}+\frac{\pi}{8 \rho}\left(1+\beta_{e}-\beta_{e} \beta_{\mathrm{i}}\right) J_{0} M_{0} e^{-\frac{\pi}{l} \tilde{y}} \\
\frac{\partial \tilde{w}}{\partial \tilde{t}}-v_{0} \frac{\partial \tilde{w}}{\partial \tilde{y}}=v \frac{\partial^{2} \tilde{w}}{\partial \tilde{y}^{2}}+\frac{\pi}{8 \rho}\left(1-\beta_{e}-\beta_{e} \beta_{\mathrm{i}}\right) J_{0} M_{0} e^{-\frac{\pi}{l} \tilde{y}} \\
\frac{\partial \tilde{T}}{\partial \tilde{t}}-v_{0} \frac{\partial \tilde{T}}{\partial \tilde{y}}=\frac{k}{\rho C_{p}} \frac{\partial^{2} \tilde{T}}{\partial \tilde{y}^{2}}+\frac{\mu}{\rho c_{p}}\left(\frac{\partial \tilde{w}}{\partial \tilde{y}}\right)^{2}
\end{gathered}
$$

The corresponding boundary conditions are

$$
\begin{aligned}
& t \leq 0: \quad u=0, \quad w=0, T=T_{1} \text { for all } y \geq 0 \\
& t>0:\left\{\begin{array}{l}
u=0, \quad w=0, T=T_{1} \text { at } y=0 \\
u=\frac{\pi v}{l}, w=0, T=T_{2} \text { at } y=d
\end{array}\right.
\end{aligned}
$$

\subsection{Similarity Analysis}

The following non-dimensional variables are used to make the dimensionless form of the equations (1) to (4).

$$
\begin{aligned}
x=\frac{\pi}{l} \tilde{x}, \quad y=\frac{\pi}{l} \tilde{y}, \quad u & =\frac{l}{\pi v} \tilde{u}, \quad w=\frac{l}{\pi v} \tilde{w}, t=\frac{\pi^{2} v}{l^{2}} \tilde{t}, \\
\theta & =\frac{\tilde{T}-\widetilde{T}_{1}}{\widetilde{T}_{2}-\widetilde{T}_{1}}
\end{aligned}
$$

Applying these into the equations (1)-(4), yields

$$
\begin{gathered}
\frac{\partial u}{\partial t}-V_{0} \frac{\partial u}{\partial y}=\alpha+\frac{\partial^{2} u}{\partial y^{2}}+H_{r}\left(1+\beta_{e}-\beta_{e} \beta_{\mathrm{i}}\right) e^{-y} \\
\frac{\partial w}{\partial t}-V_{0} \frac{\partial w}{\partial y}=\frac{\partial^{2} w}{\partial y^{2}}+H_{r}\left(1-\beta_{e}-\beta_{e} \beta_{\mathrm{i}}\right) e^{-y} \\
\frac{\partial \theta}{\partial t}-V_{0} \frac{\partial \theta}{\partial y}=\frac{1}{P_{r}} \frac{\partial^{2} \theta}{\partial y^{2}}-E_{c}\left(\frac{\partial w}{\partial y}\right)^{2}
\end{gathered}
$$

The corresponding boundary conditions are

$$
\begin{aligned}
& t \leq 0: u=0, \theta=0 \text {, for all } y \geq 0 \\
& t>0: \begin{cases}u=0, & w=0, \quad \theta=0 \text { at } y=0 \\
u=1, & w=0, \theta \rightarrow 1, \text { at } y \rightarrow d\end{cases}
\end{aligned}
$$

where, $\quad \alpha=-\frac{l^{3} \mathrm{P}}{\rho v^{2} \pi^{3}}$ is the dimensionless pressure gradient, $\quad H_{r}=\frac{l^{3} J_{0} M_{0}}{8 \rho v^{2} \pi^{2}}$ is the modified Hartmann Number; $\quad P_{r}=\frac{\rho c_{p} v}{k}$ is the Prandtl number ; $E_{c}=\frac{v^{2} \pi^{2}}{\left(\widetilde{T}_{2}-\widetilde{T}_{1}\right) c_{p} l^{2}}$ is the Eckert Number.

\section{Method of Solution}

We apply the explicit finite difference method to solve the non-dimensional coupled partial differential equations (5)-(7) with the associated boundary conditions (8).

It is considered maximum length of the plate is $\left.x_{\max }=10\right)$ and distance between the plates $d=2$ i.e. $y_{\max }=2$ as the lower plate is fixed at $y=0$. This means $x$ varies from 0 to 10 and $y$ varies from 0 to 2 . The finite difference schemes for the problems are as follows:

$$
\begin{gathered}
\frac{u_{i, j}^{k+1}-u_{i, j}^{k}}{\Delta t}-V_{0} \frac{u_{i, j}^{k}-u_{i, j-1}^{k}}{\Delta y}=\alpha+\frac{u_{i, j+1}^{k}-2 u_{i, j}^{k}+u_{i, j-1}^{k}}{\Delta y^{2}} \\
+H_{r}\left(1+\beta_{e}-\beta_{e} \beta_{\mathrm{i}}\right) e^{-y} i_{i} \\
\frac{w_{i, j}^{k+1}-w_{i, j}^{k}}{\Delta t}-V_{0} \frac{w_{i, j}^{k}-w_{i, j-1}^{k}}{l}=\frac{w_{i, j+1}^{k}-2 w_{i, j}^{k}+w_{i, j-1}^{k}}{l^{2}} \\
+H_{r}\left(1-\beta_{e}-\beta_{e} \beta_{\mathrm{i}}\right) e^{-y_{i}} \\
\frac{\Theta_{i, j}^{k+1}-\Theta_{i, j}^{k}}{\Delta t}-V_{0} \frac{\Theta_{i, j}^{k}-\Theta_{i, j-1}^{k}}{\Delta y}=\frac{1}{P_{r}}\left(\frac{\Theta_{i, j+1}^{k}-2 \Theta_{i, j}^{k}+\Theta_{i, j-1}^{k}}{\Delta y^{2}}\right) \\
-E_{c} \frac{w_{i, j}^{k}-w_{i, j-1}^{k}}{\Delta y}
\end{gathered}
$$


with the boundary conditions

$$
\begin{aligned}
& t \leq 0: \quad u=0, \quad \theta=0, \text { for all } y \geq 0 \\
& t>0: \begin{cases}u=0, & w=0, \quad \theta=0 \text { at } y=0 \\
u=1, & w=0, \theta=1, \text { at } y=d\end{cases}
\end{aligned}
$$

Here, the subscripts $\mathrm{i}$ and $\mathrm{j}$ refer to $\mathrm{x}$ and $\mathrm{y}$ and the superscript $\mathrm{k}$ refers to time $\mathrm{t}$.

\subsection{Shear Stresses and Nusselt Number}

The effects of pertinent parameters on the local and average shear stress from the velocity of the fluid have been investigated. The non-dimensional form of the local and average shear stress for the fluid is given by the relations $\tau_{L}=\left.\mu \frac{\partial u}{\partial y}\right|_{y=0}$ and $\tau_{A}=\left.\frac{1}{L} \int_{0}^{L} \mu \frac{\partial u}{\partial y}\right|_{y=0} d x$ and for the dust particle are given by $\tau_{p L}=\left.\mu \frac{\partial u_{p}}{\partial y}\right|_{y=0}$ and $\tau_{p A}=\left.\frac{1}{L} \int_{0}^{L} \mu \frac{\partial u_{p}}{\partial y}\right|_{y=0} d x$

respectively. The rate of heat transfer at the plate is defined as the Nusselt number. The local and average Nusselt number for the fluid are given by $N u_{L}=-\left.\mu \frac{\partial \theta}{\partial y}\right|_{y=0}$ and $N u_{A}=-\left.\frac{1}{L} \int_{0}^{L} \mu \frac{\partial \theta}{\partial y}\right|_{y=0} d x$.

\subsection{Steady-state Solution}

The behavior of the various entities on the velocity and temperature profiles has been elaborated graphically. Fig.2(a), Fig.2(b), and Fig.2(c) illustrate the fluid velocities and temperature distributions $u, w$ and $\theta$ for different time $\tau$. The computations have been carried out for the different time such as $\tau=1,3,6$ and 8 with the time step size $\Delta t=0.0005$. It is observed from figures that there is no change after $\tau=8$ for $U, w$ and $\theta$. Fig.2(d) and Fig.2(e) display the validity of the grid pairs on the velocity $u$ and temperature $\theta$. It has shown the velocity and temperature distributions for three grid pairs $(m, n)=(40,40), \quad(m, n)=(45,45)$ and $(m, n)=(50,50) \quad$ with time $\tau=8$ and time step $\Delta t=0.0005$. There is also negligible change among these grid pairs so that any one grid pair is acceptable to find the steady-state solution. It has been seen that the same situation occurs for the other distributions. The steady-state solution has been performed for the values of $\tau>7$. In the present analysis, the following graphs have been established for the choice of time $\tau=8$ with the grid pair $(m, n)=(50,50)$ and time step $\Delta t=0.0005$.

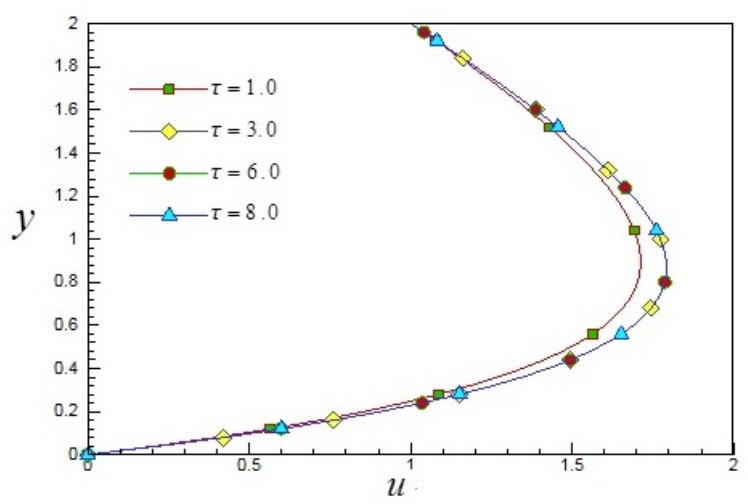

Figure 2(a). Time sensitivity on primary velocity $u$

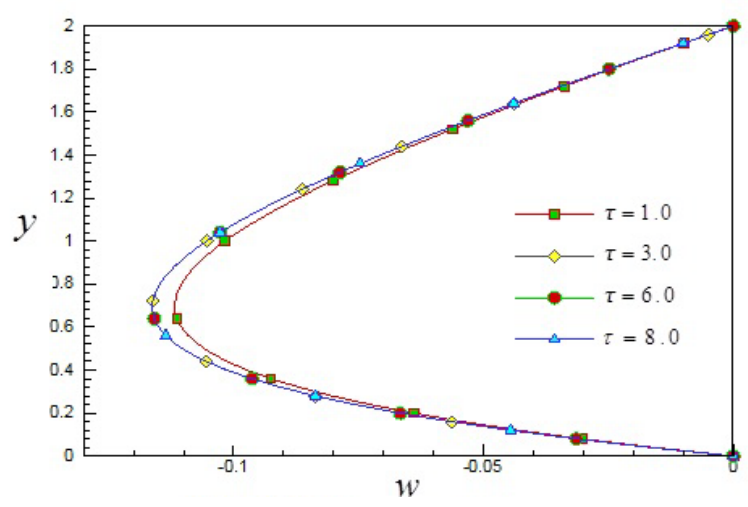

Figure 2(b). Time sensitivity on secondary velocity $w$

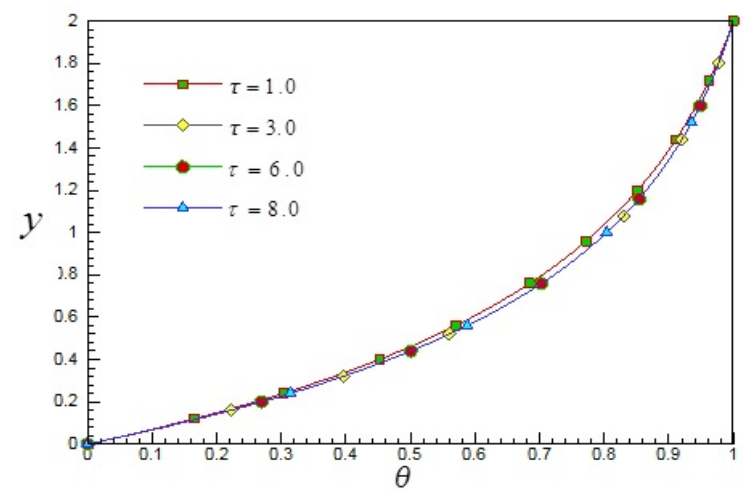

Figure 2(c). Time sensitivity on temperature profile $\theta$

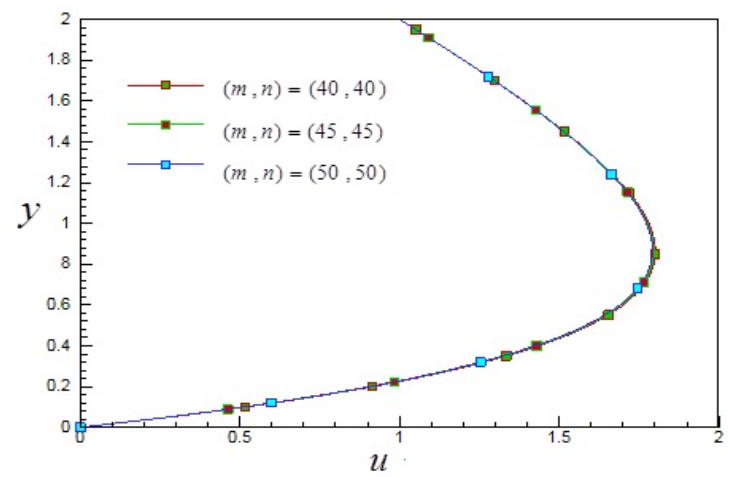

Figure 2(d). Mesh sensitivity on primary velocity $u$ 


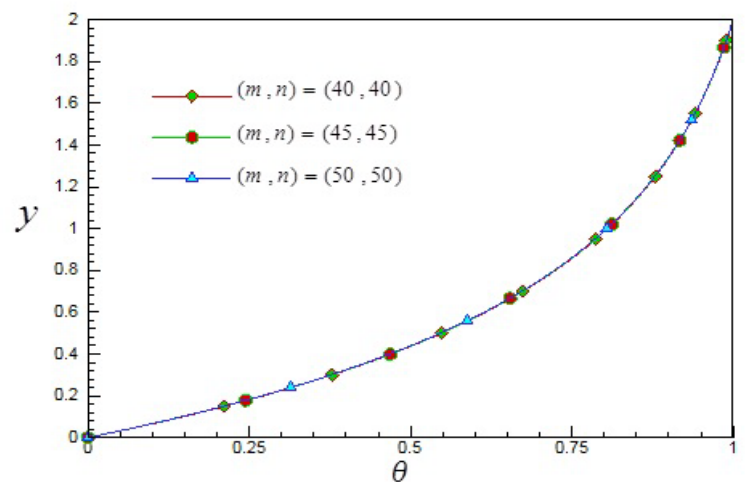

Figure 2(e). Mesh sensitivity on temperature profile $\theta$

\subsection{Effects of Various Parameters}

To study the physical situation of the problem, it is mentioned that the figures (a), (b) , and (c) of Fig.3 to Fig.7 presented the distribution of velocity, local shear stress and average shear stress along the $\mathrm{x}$-direction respectively. Figures (a), (b), and (c) of Fig.8 to Fig.11 depicted the distribution of velocity, local shear stress and average shear stress along y-direction respectively. And figures (a), (b), and (c) of Fig.12 to Fig.17 represented the distribution of temperature, local Nusselt number, and average Nusselt number respectively.

The effects of dimensionless pressure gradient $\alpha$ on the velocity is shown in Figs.3(a)-(c). Since $\alpha=-l^{3} \mathrm{P} /\left(\rho v^{2} \pi^{3}\right)$, it means that $\alpha>0$ whenever a constant pressure gradient $\mathrm{P}$ decreasing in the direction of motion; in that case, the velocity $u$ has an increasing effect with the increase of $\alpha$ over the entire width between the plates. But $\alpha<0$ influence that $\mathrm{P}$ is increasing in the direction of motion and the velocity $u$ has occurred back-flow, which been shown in Fig.3(a). The same situation occurred for the local and average shear stress on the fluid which have shown in Figs.3(b) and (c) respectively.

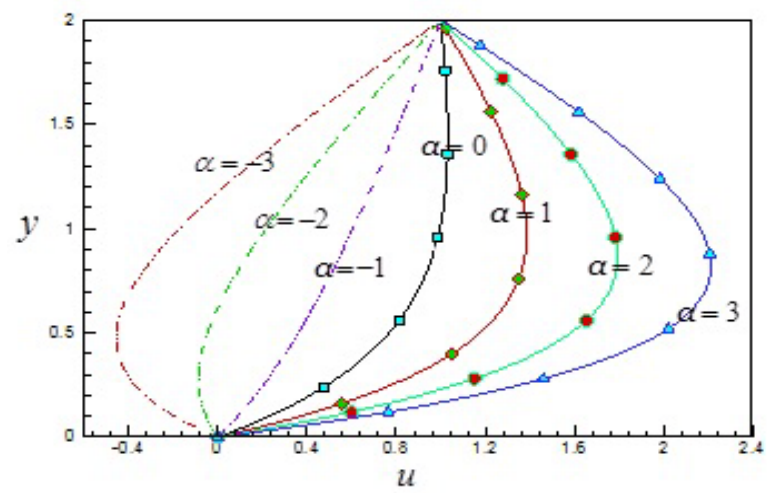

Figure 3(a). Effect of pressure gradient $\alpha$ on primary velocity

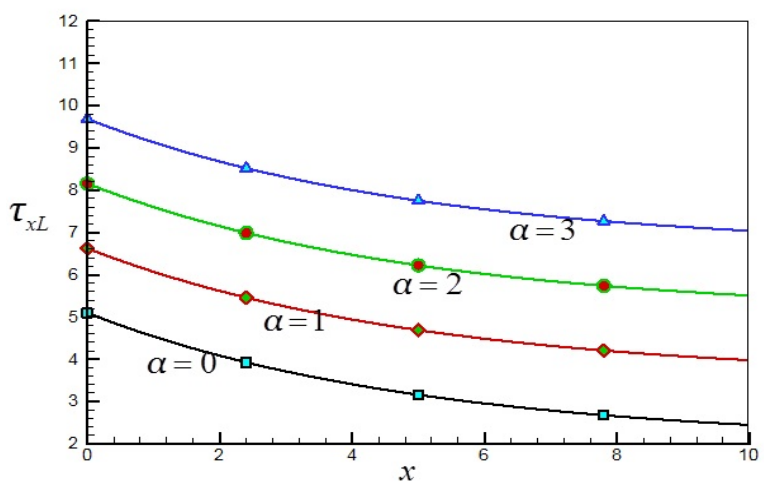

Figure 3(b). Effect of pressure gradient $\alpha$ on local shear stress $\tau_{x L}$

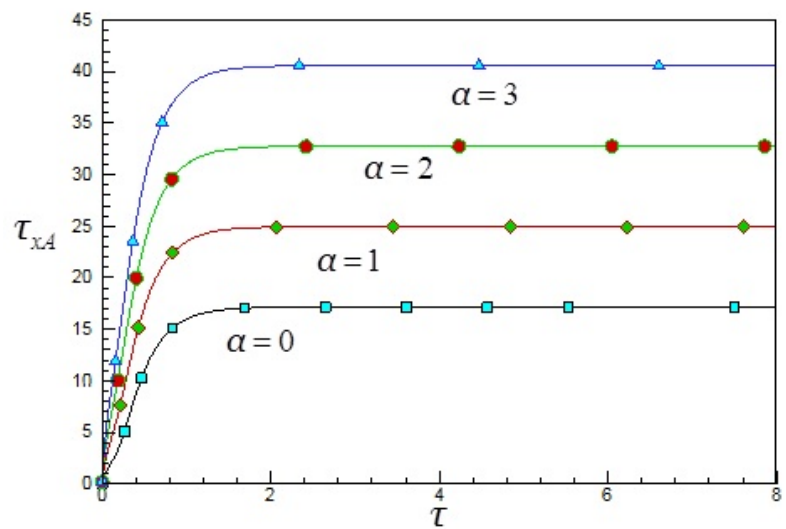

Figure 3(c). Effect of pressure gradient $\alpha$ on average shear stress

In the figures, Figs.4(a)-(c) represented the performance of Hall parameter $\beta_{e}$ on the primary velocity $u$, local shear stress $\tau_{x L}$ and on the average shear stress $\tau_{x A}$ respectively.

It is showed that the primary velocity $u$, local and average shear stress $\tau_{x L}$ and $\tau_{x A}$ are all increasing with the increasing values of Hall current $\beta_{e}$

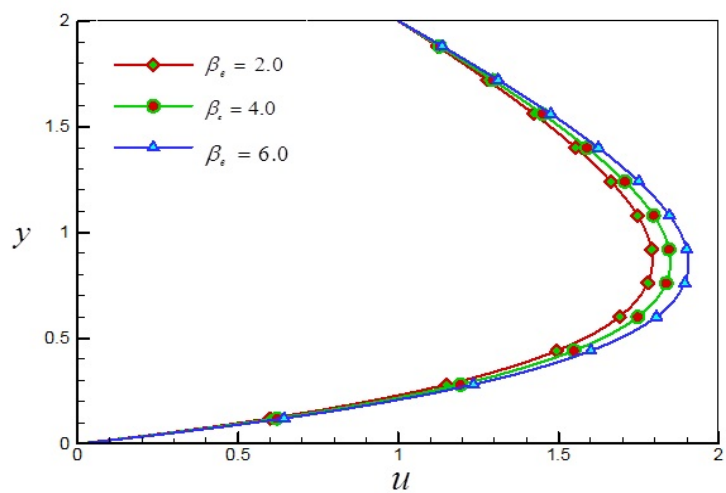

Figure 4(a). Effect of Hall current $\beta_{e}$ on primary velocity $u$ 


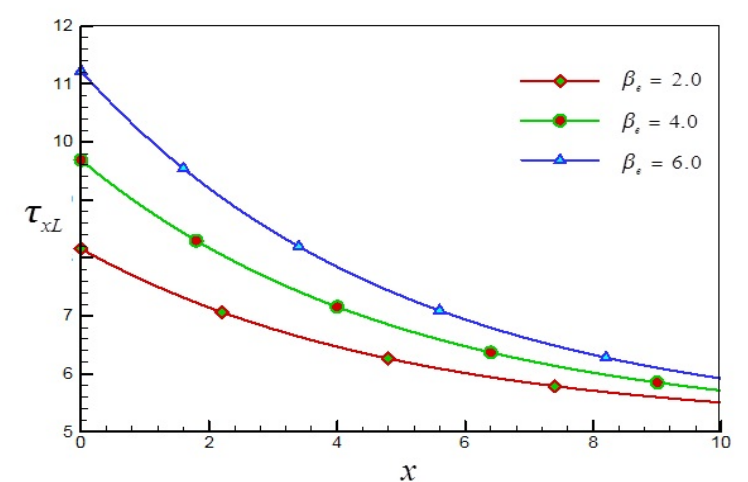

Figure 4(b). Effect of Hall current $\beta_{e}$ on local shear stress $\tau_{x L}$

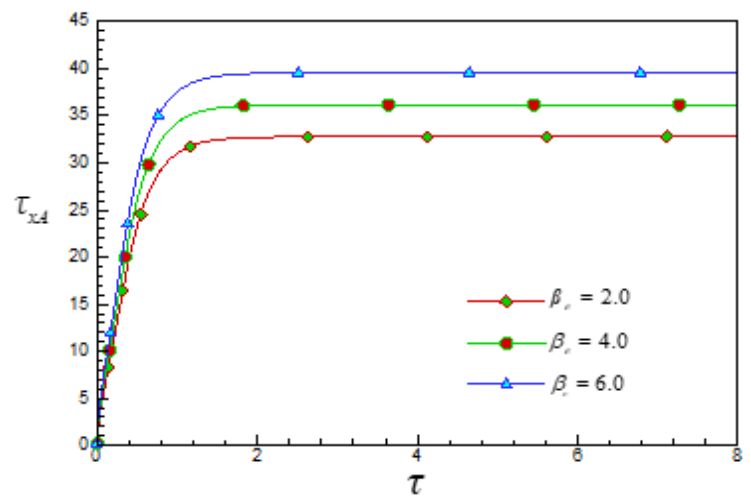

Figure 4(c). Effect of Hall current $\beta_{e}$ on average shear stress $\tau_{x A}$

The impact of Ion-slip parameter $\beta_{i}$ on the primary velocity $u$, local shear stress $\tau_{x L}$ and the average shear $\tau_{x A}$ stress are displayed respectively in Figs.5(a)-(c). It is observed that the primary velocity $u$, local and average shear stress $\tau_{x L}$ and $\tau_{x A}$ all are decreased with the increase of $\beta_{i}$.

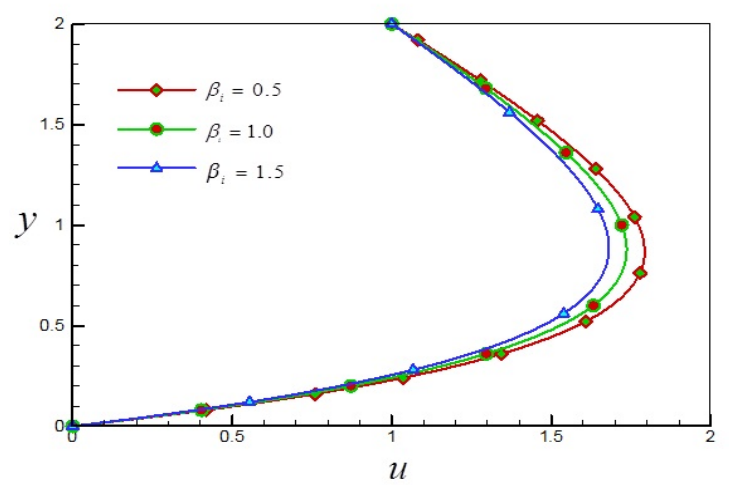

Figure 5(a). Effect of Ion-slip $\beta_{i}$ on primary velocity $\boldsymbol{u}$

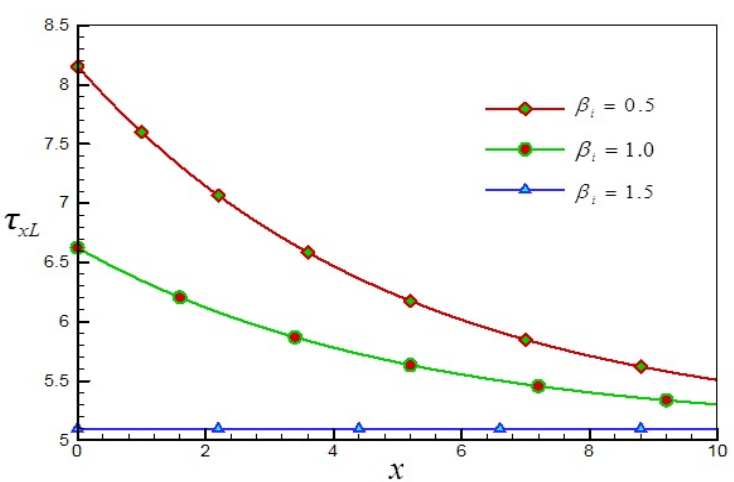

Figure 5(b). Effect of Ion-slip $\beta_{i}$ on local shear stress $\tau_{x L}$

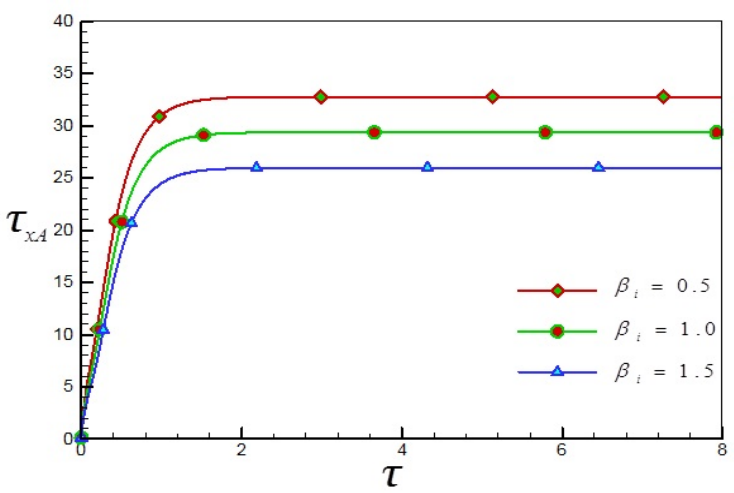

Figure 5(c). Effect of Ion-slip $\beta_{i}$ on average shear stress $\tau_{x A}$

Figs.6(a)-(c) depict that the effect of modified Hartmann number $H_{r}$ on the primary velocity $u$, local shear stress $\tau_{x L}$ and the average shear stress $\tau_{x A}$ respectively. It is evident that $u, \tau_{x L}$ and $\tau_{x A}$ are all rises with the influence of $H_{r}$.

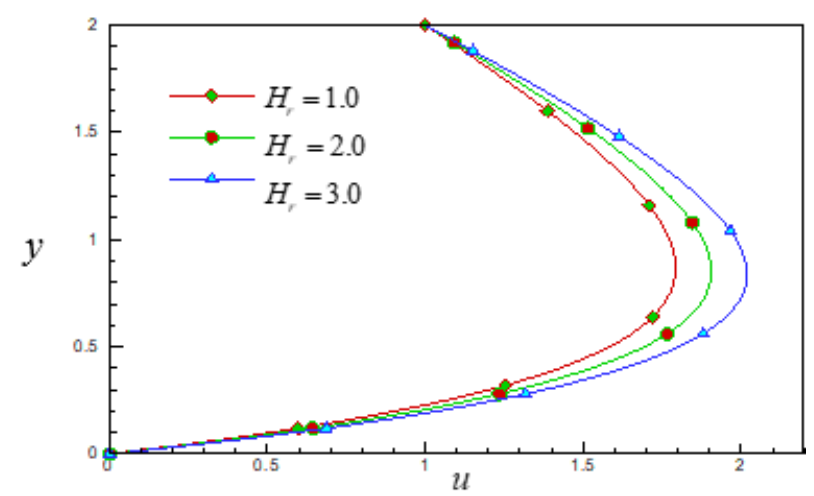

Figure 6(a). Effect of modified Hartmann number $H_{r}$ on primary velocity $\boldsymbol{u}$ 


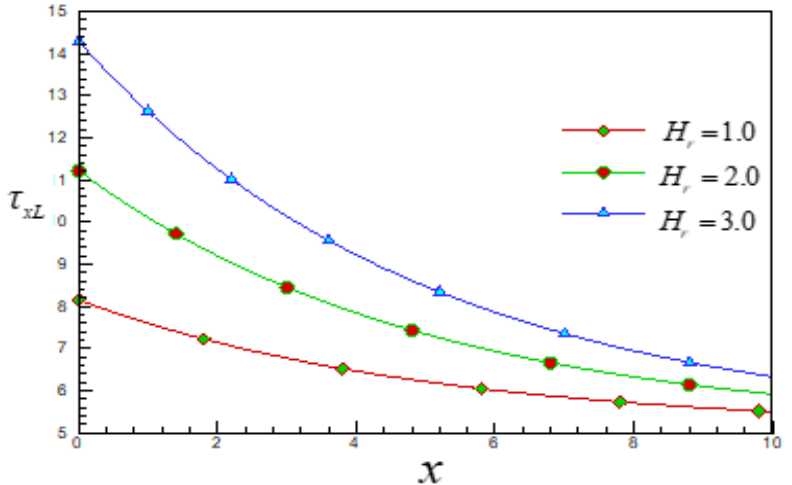

Figure 6(b). Effect of modified Hartmann number $H_{r}$ on local shear stress $\tau_{x L}$

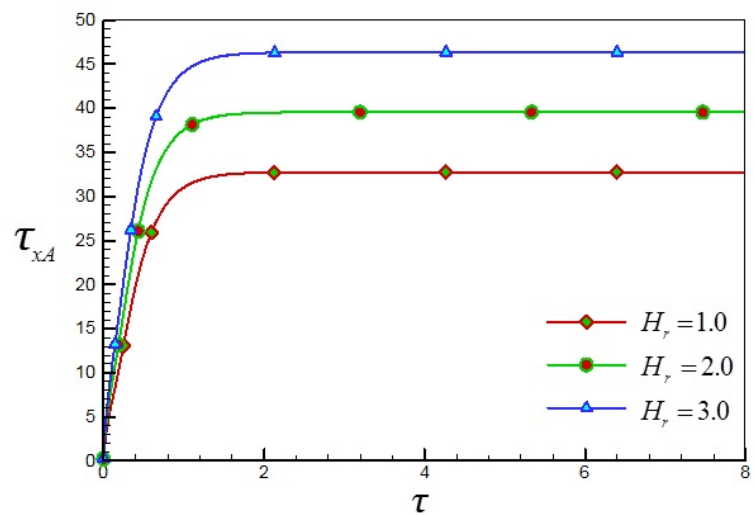

Figure 6(c). Effect of modified Hartmann number $H_{r}$ on average shear stress $\tau_{x A}$

Characteristics of the Suction velocity $V_{0}$ on the primary velocity $u$, local shear stress $\tau_{x L}$ and the average shear stress $\tau_{x A}$ are presented in Figs.7(a)-(c) respectively. In Fig.7(a), it is observed that the velocity $\boldsymbol{u}$ has a cross flow where the velocity has an increasing effect within the interval $0<y<1$ (approx.) thereafter it has a decreasing effect, whereas $\tau_{x L}$ and $\tau_{x A}$ are increased with the increasing effect of $V_{0}$.

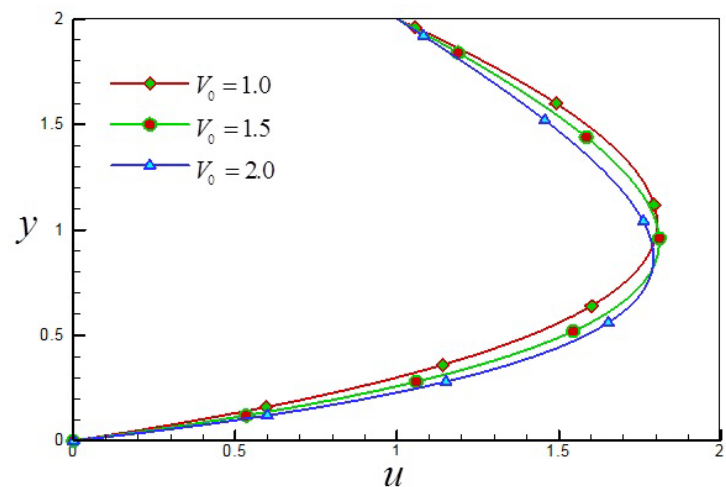

Figure 7(a). Effect of Suction velocity $V_{0}$ on primary velocity $u$

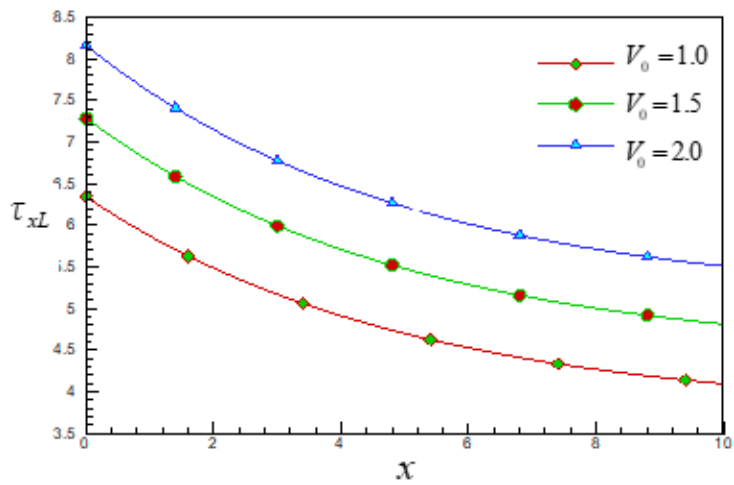

Figure 7(b). Effect of Suction velocity $V_{0}$ on local shear stress $\tau_{x L}$

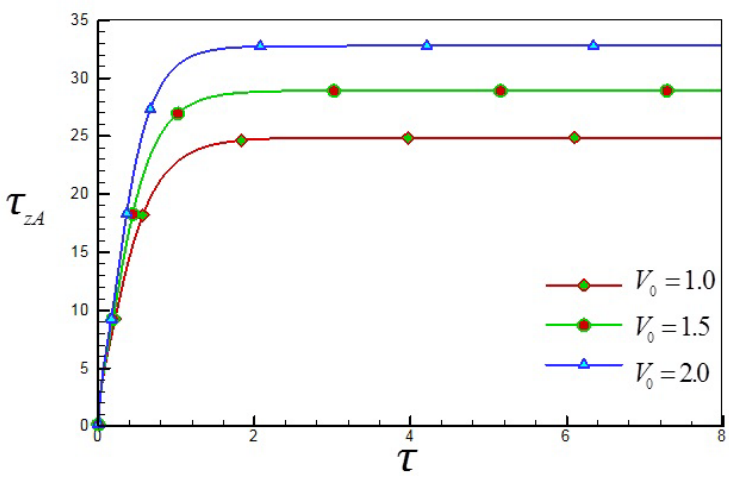

Figure 7(c). Effect of Suction velocity $V_{0}$ on average shear stress $\tau_{x A}$

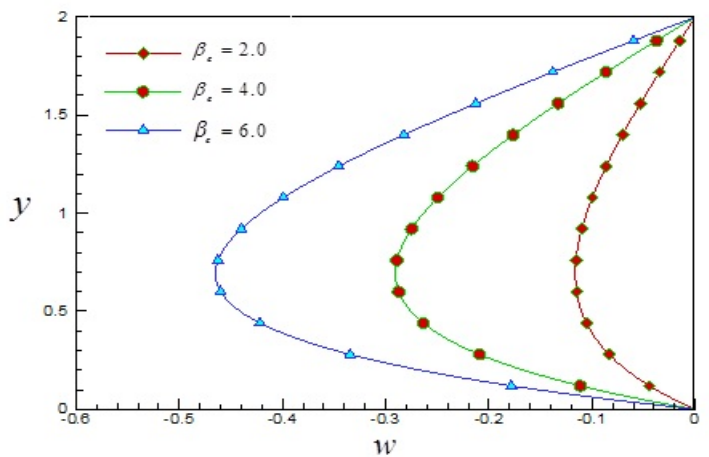

Figure 8(a). Effect of Hall current $\beta_{e}$ on secondary velocity $w$

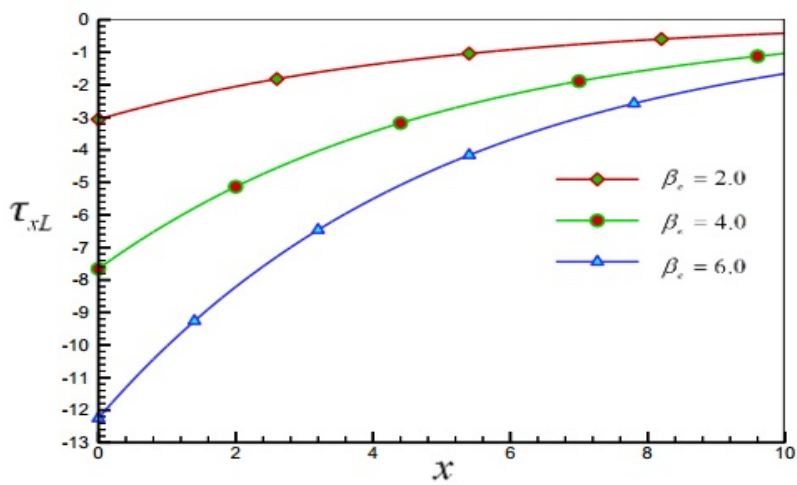

Figure 8(b). Effect of Hall current $\beta_{e}$ on local shear stress $\tau_{x L}$ 


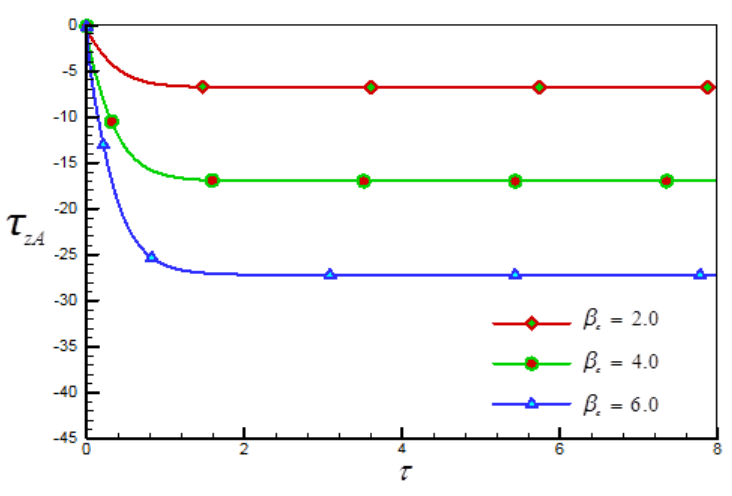

Figure 8(c). Effect of Hall current $\beta_{e}$ on average shear stress $\tau_{\mathrm{zA}}$

Figs.8(a)-(c) explain the influence of Hall current parameter $\beta_{e}$ on the secondary velocity $w$, local shear stress $\tau_{z L}$ and the average shear stress $\tau_{z A}$ respectively. It has shown that the increasing values of $\beta_{e}$ the secondary velocity $w$, local shear stress $\tau_{z L}$ and average shear stress $\tau_{z A}$ become decreases.

The effects of Ion-slip parameter $\beta_{i}$ on the secondary velocity profile $w$, local shear stress $\tau_{z L}$ and the average shear stress $\tau_{z \mathrm{~A}}$ are exposed in Figs.9(a)-(c) respectively. It has shown for the increasing values of $\beta_{i}$, the secondary velocity profile $w$, local and average shear stress $\tau_{z L}$ and $\tau_{z A}$ are decreased.

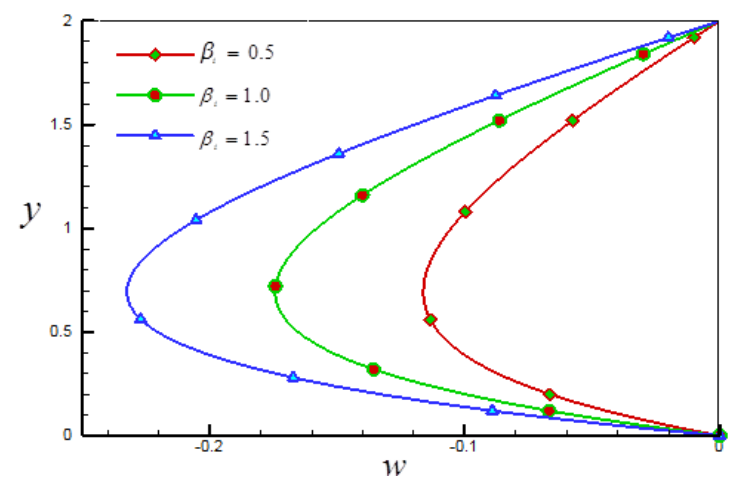

Figure 9(a). Effect of Ion-slip $\beta_{i}$ on secondary velocity profile $w$

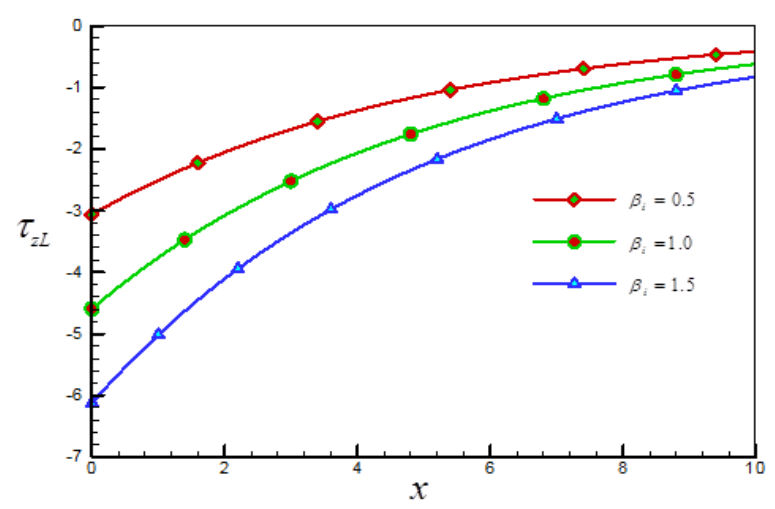

Figure 9(b). Effect of Ion-slip $\beta_{i}$ on local shear stress $\tau_{z L}$

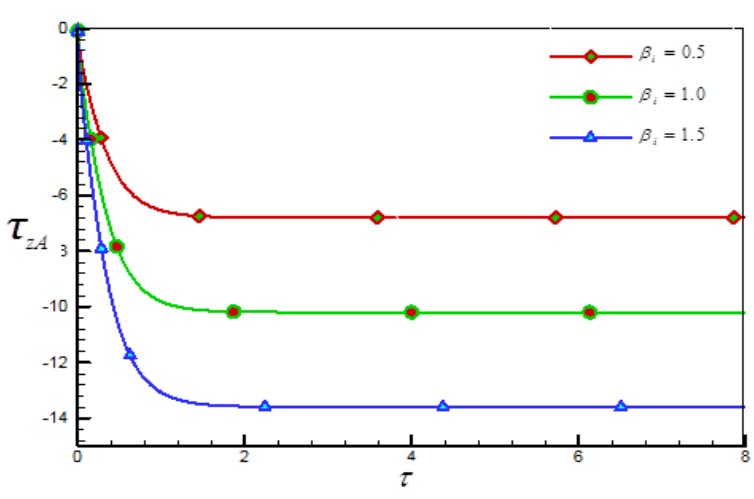

Figure 9(c). Effect of Ion-slip $\beta_{i}$ on average shear stress $\tau_{\text {zA }}$

The variations of modified Hartmann number $H_{r}$ on the secondary velocity $w$, local shear stress $\tau_{z L}$ and on the average shear stress $\tau_{z A}$ are depicted in Figs.10(a)-(c) . With the increasing effect of $H_{r}$ it has found that the secondary velocity $w$, local and average shear stress $\tau_{z L}$ and $\tau_{z \mathrm{~A}}$ are decreased.

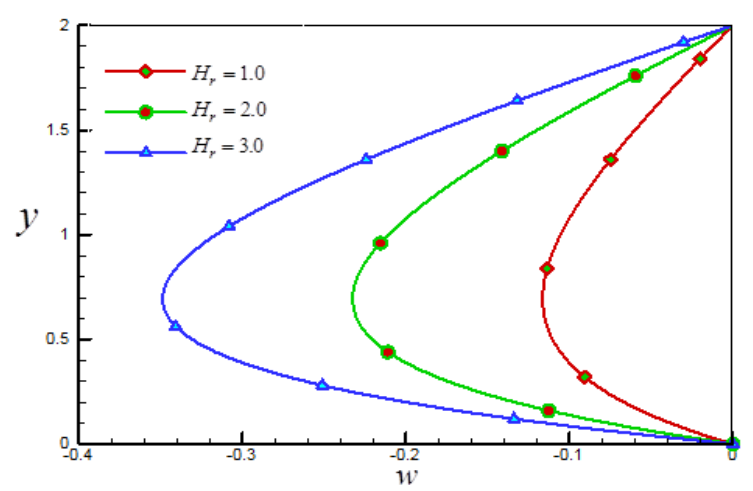

Figure 10(a). Effect of modified Hartmann number $H_{r}$ on secondary velocity $W$

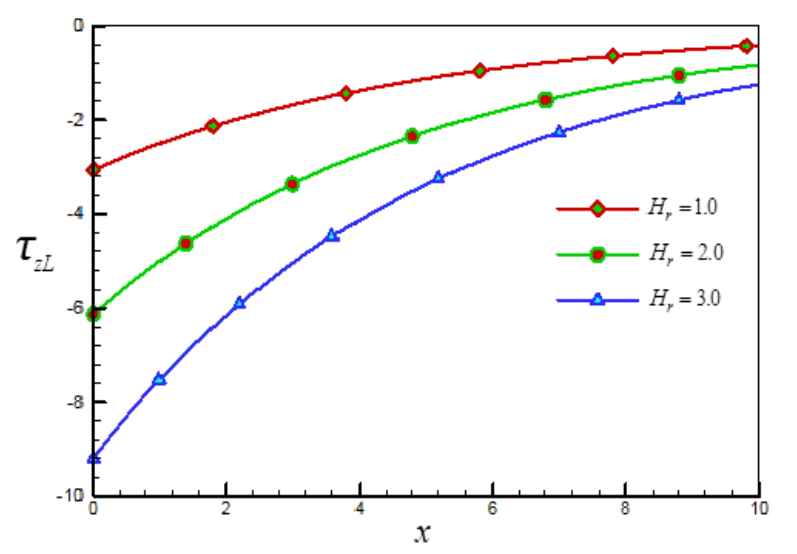

Figure 10(b). Effect of modified Hartmann number $H_{r}$ on local shear stress $\tau_{z L}$ 


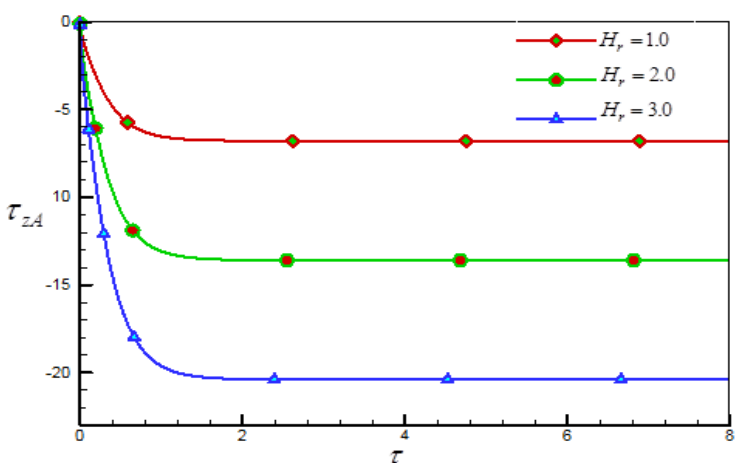

Figure 10(c). Effect of modified Hartmann number $H_{r}$ on average shear stress $\tau_{z A}$

Figs.11(a)-(c) are plotted to explain the behavior of suction velocity $V_{0}$ on the secondary velocity $\mathrm{w}$, local shear stress $\tau_{z L}$ and the average shear stress $\tau_{z A}$ respectively. It has shown that the back flow of $w$ with the increasing values of suction velocity $V_{0}$, whereas $\tau_{z L}$ and $\tau_{z \mathrm{~A}}$ are displayed decreasing effect.

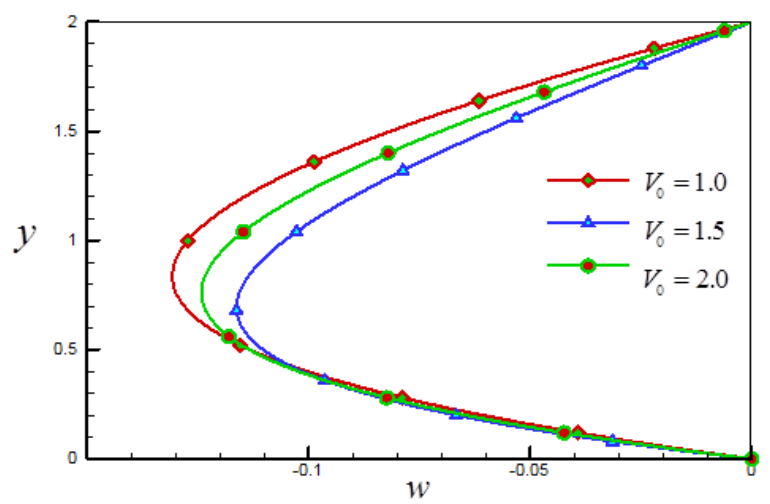

Figure 11(a). Effect of suction velocity $V_{\mathrm{o}}$ on secondary velocity $W$

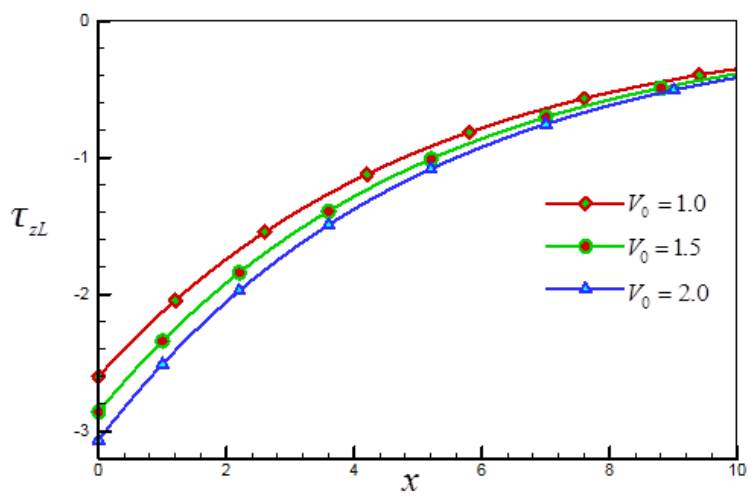

Figure 11(b). Effect of suction velocity $V_{0}$ on local shear stress $\tau_{z L}$

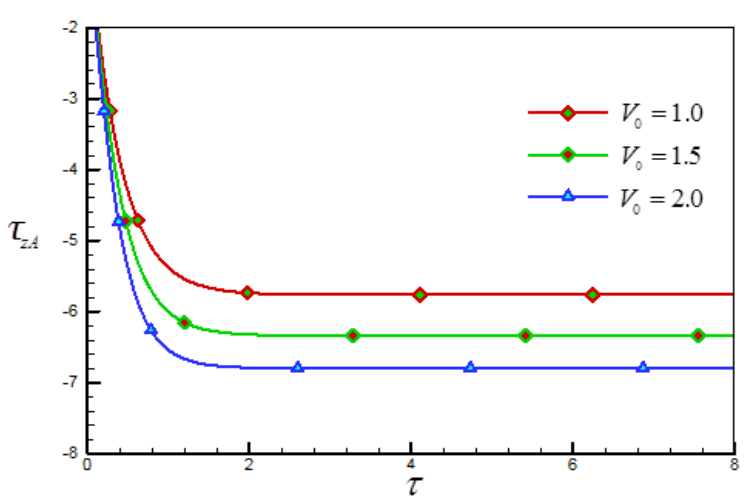

Figure 11(c). Effect of suction velocity $V_{0}$ on average shear stress $\tau_{\mathrm{zA}}$

To find the variations of Hall current parameter $\beta_{e}$ on the temperature $\theta$, local Nusselt number $N u_{L}$ and the average Nusselt number $N u_{A}$ have been demonstrated in Figs.12(a)-(c) respectively. It is found from the figures that for the increasing effect of $\beta_{e}$, temperature profile $\theta$ is increased whereas $N u_{L}$ and $N u_{A}$ showed the inverse effects.

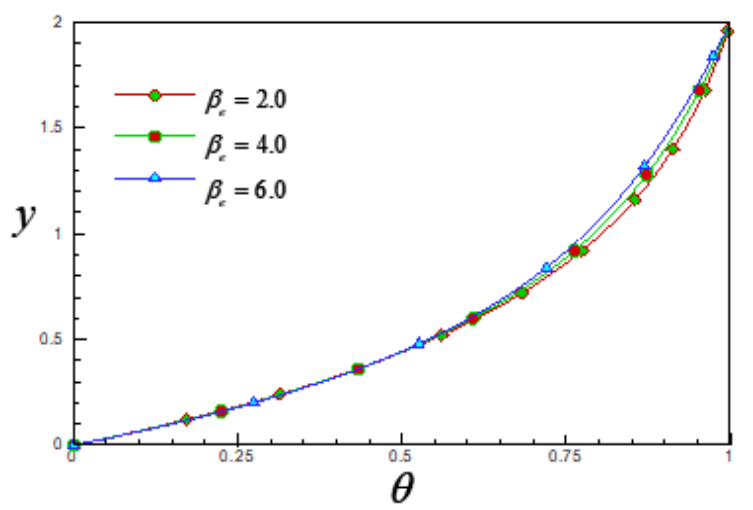

Figure 12(a). Effect of Hall current $\beta_{e}$ on temperature profile $\theta$

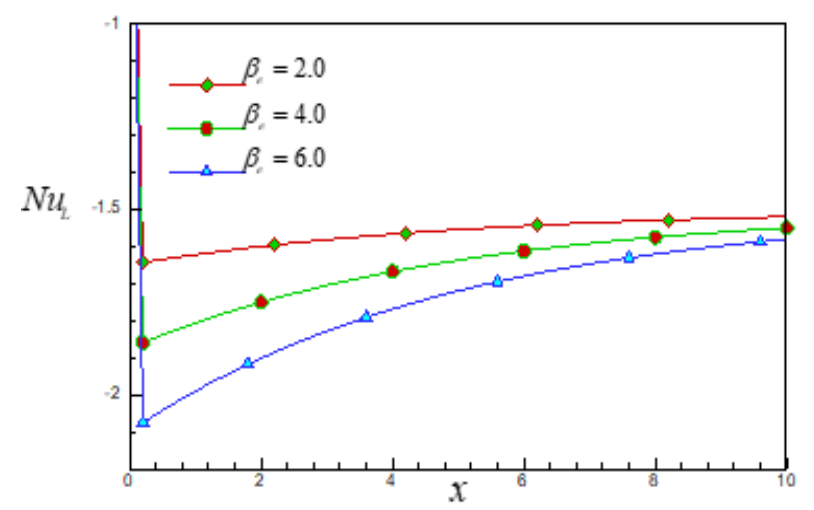

Figure 12(b). Effect of Hall current $\beta_{e}$ on local Nusselt number $N u_{L}$ 


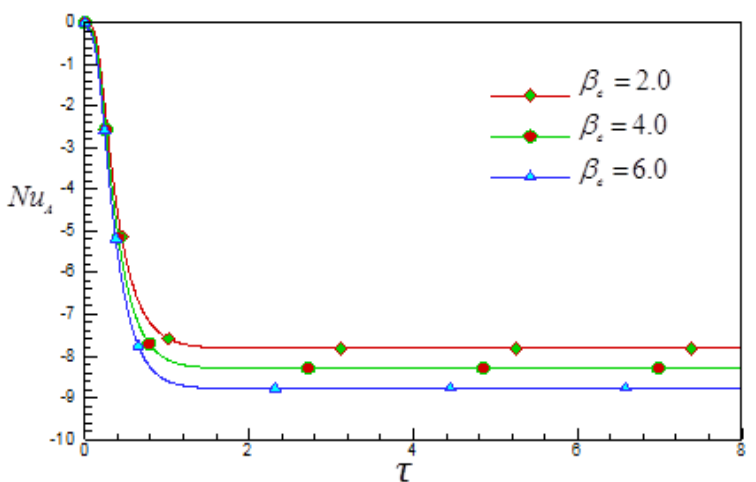

Figure 12(c). Effect of Hall current $\beta_{e}$ on average Nusselt number $\mathrm{Nu}{ }_{\mathrm{A}}$

The effects of Ion-slip current parameter $\beta_{i}$ on the temperature $\theta$, local Nusselt number $N u_{L}$ and the average Nusselt number $N u_{A}$ are plotted in Figs.13(a)-(c) respectively. It has shown that for the increasing effect of $\beta_{i}$, temperature $\theta$ is increased whereas $N u_{L}$ and $\mathrm{Nu}{ }_{\text {A }}$ showed the decreasing effects.

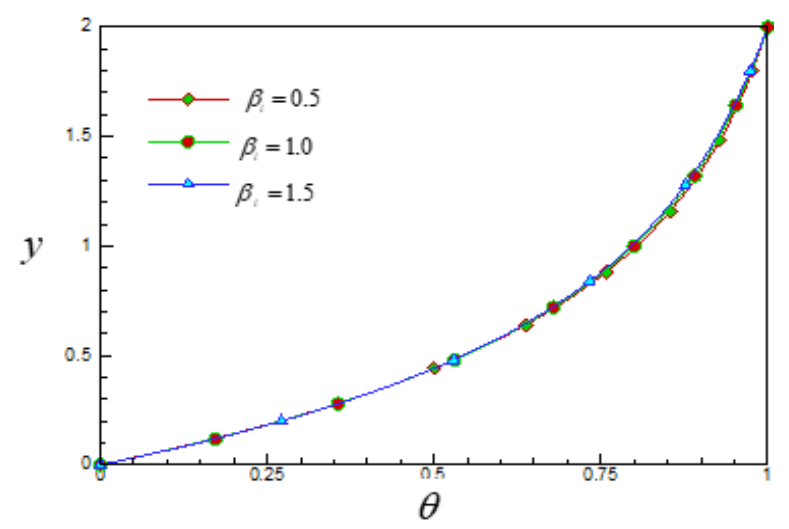

Figure 13(a). Effect of Ion-slip $\beta_{i}$ on temperature profile $\theta$

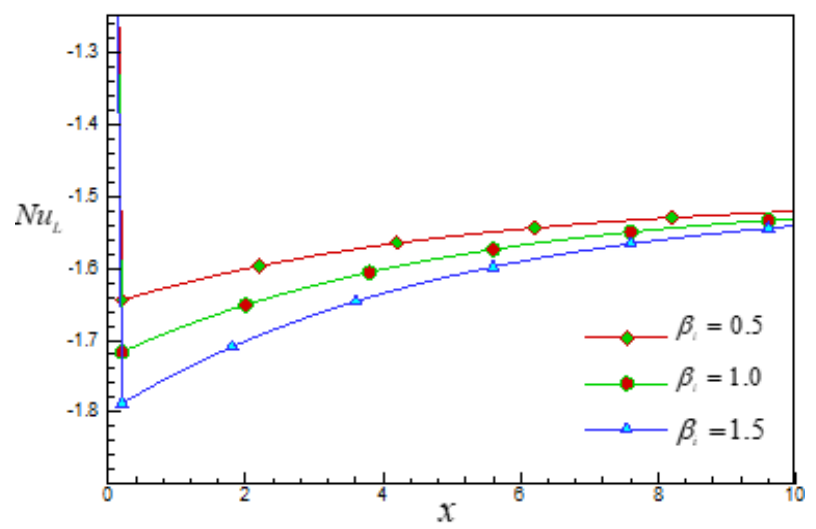

Figure 13(b). Effect of Ion-slip $\beta_{i}$ on local Nusselt number $N u_{L}$

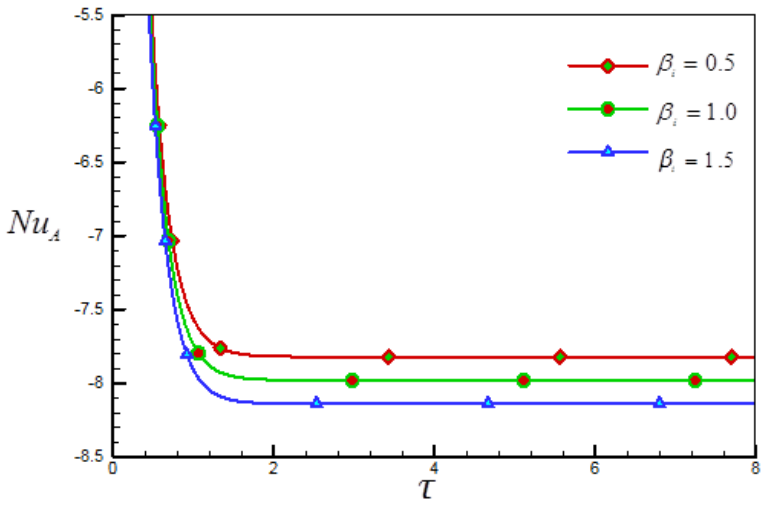

Figure 13(c). Effect of Ion-slip $\beta_{i}$ on average Nusselt number $N u_{A}$

It is visualized from the figures Figs.14(a)-(c) that for increasing values of modified Hartmann number $H_{r}$ on the temperature $\theta$ depicted rising effect and the local Nusselt number $N u_{L}$ and the average Nusselt number $N u_{A}$ are demonstrated decreasing effects.

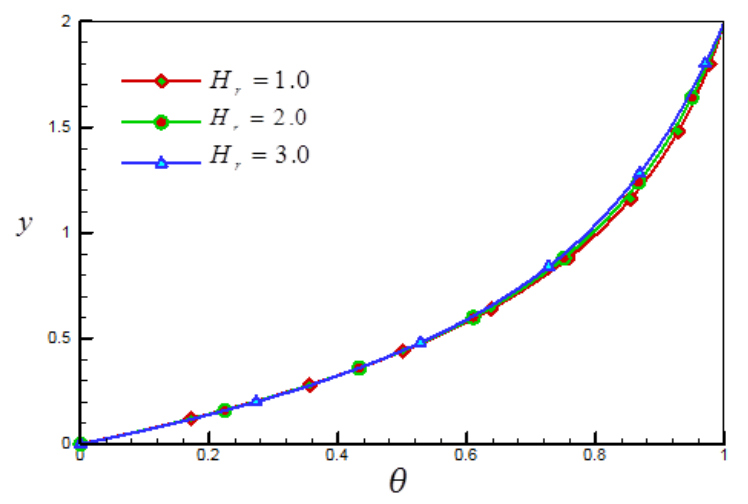

Figure 14(a). Effect of modified Hartmann number $H_{r}$ on temperature profile $\theta$

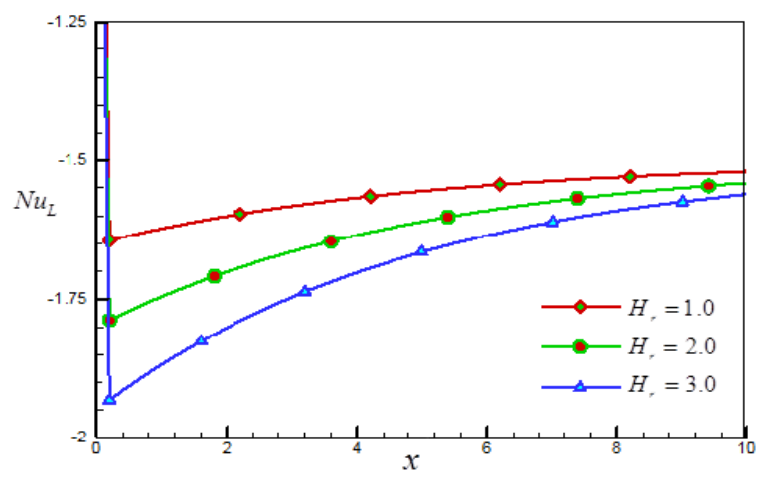

Figure 14(b). Effect of modified Hartman number $H_{r}$ on local Nusselt number $\mathrm{Nu}{ }_{L}$ 


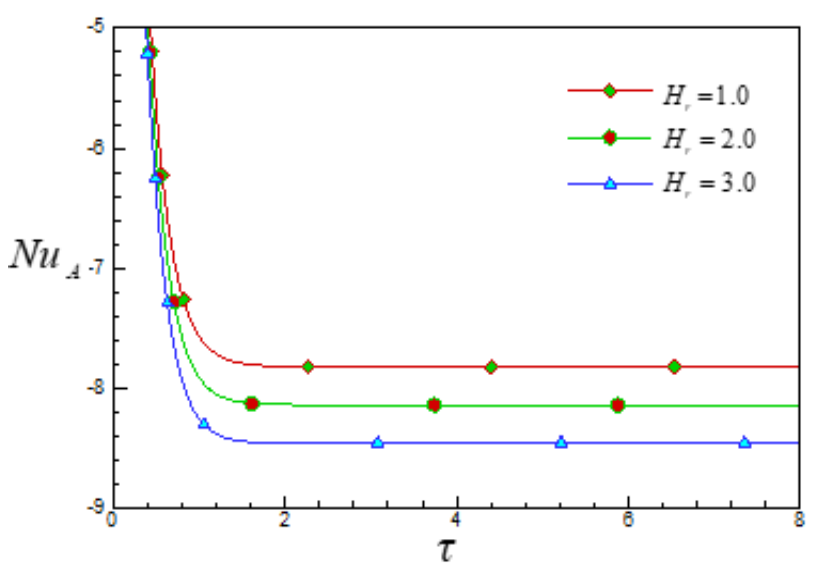

Figure 14(c). Effect of modified Hartman number $H_{r}$ on average Nusselt number $\mathrm{Nu}_{\mathrm{A}}$

Temperature profile $\theta$ is increased due to the increasing effect of the Prandtl number $\boldsymbol{P}_{r}$ that has been seen in Figs.15(a) whereas Figs.15 (b) and Figs.15 (b) exhibit the reverse effect of $\theta$.

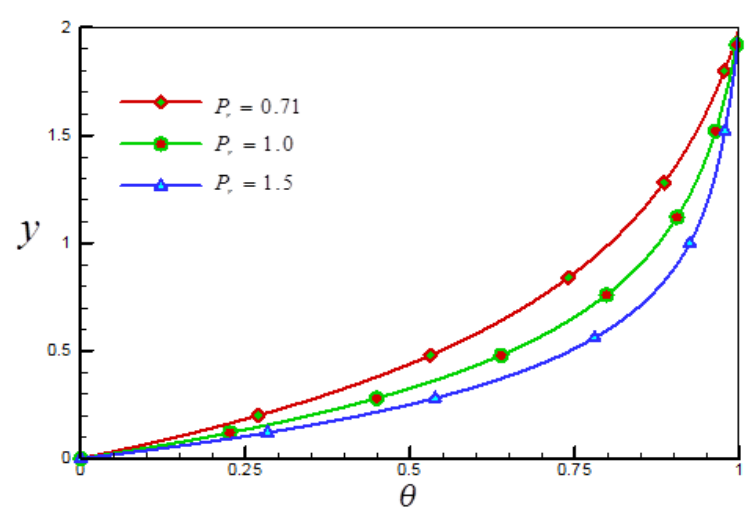

Figure 15(a). Effect of Prandtl number $P_{r}$ on temperature profile $\theta$

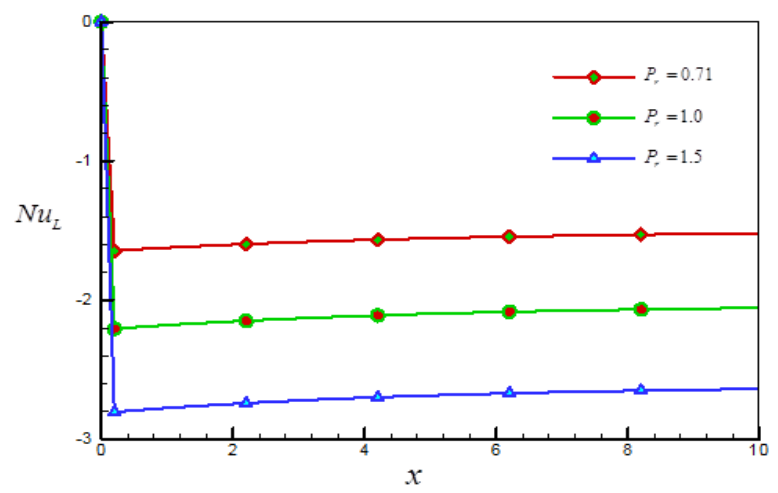

Figure 15(b). Effect of Prandtl number $P_{r}$ on local Nusselt number $\mathrm{Nu}$

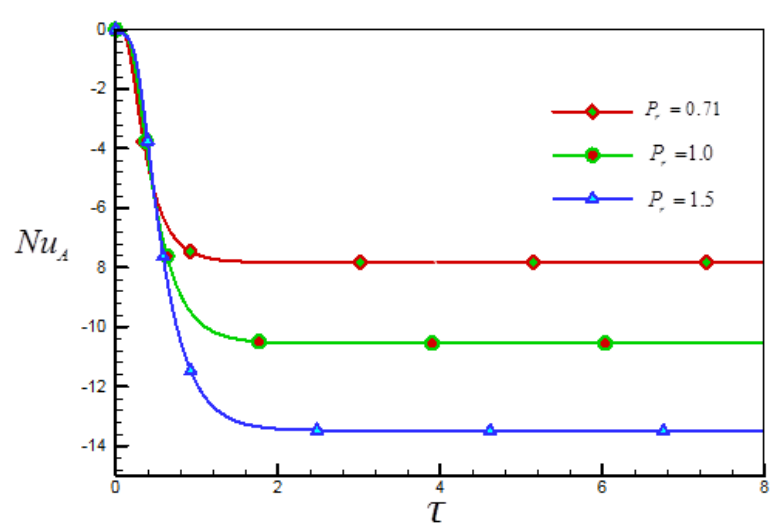

Figure 15(c). Effect of Prandtl number $P_{r}$ on average Nusselt number $N u_{A}$

It is observed from Figs.16(a)-(c) the effects of Eckert number $E_{c}$ on the temperature profile $\theta$, local Nusselt number $N u_{L}$ and the average Nusselt number $N u_{L}$ respectively. It has shown that $\theta$ depicts the increasing effect but $N u_{L}$ and $N u_{L}$ show the decreasing effect with the effects of Eckert number.

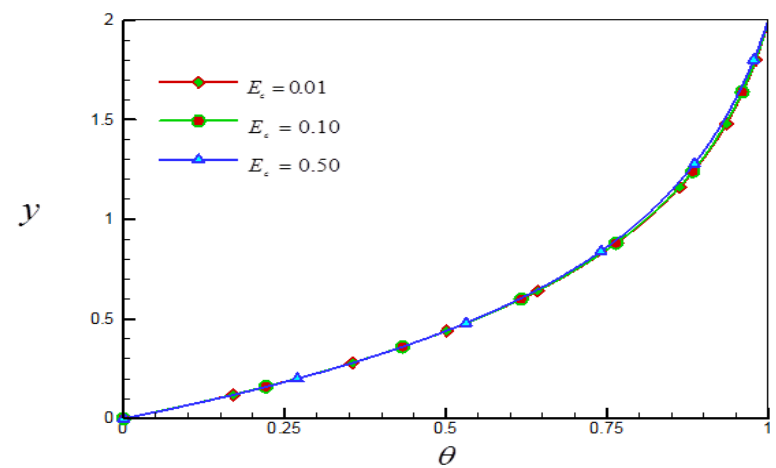

Figure 16(a). Effect of Eckert number $E_{c}$ on temperature profile $\theta$

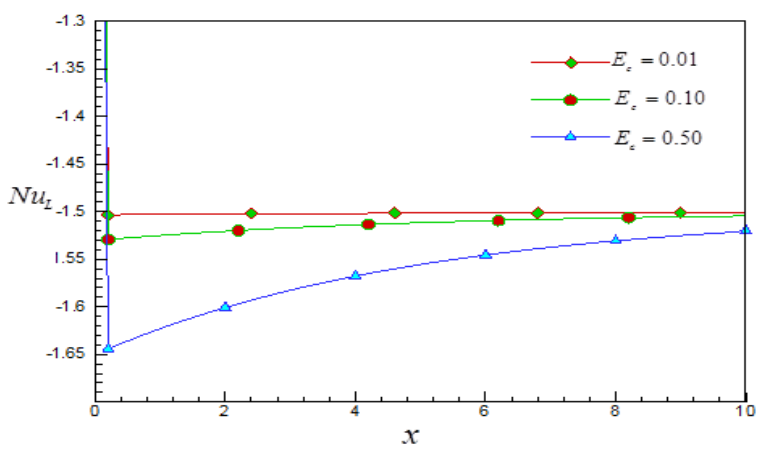

Figure 16(b). Effect of Eckert number $E_{c}$ on local Nusselt number $\mathrm{Nu}$ 


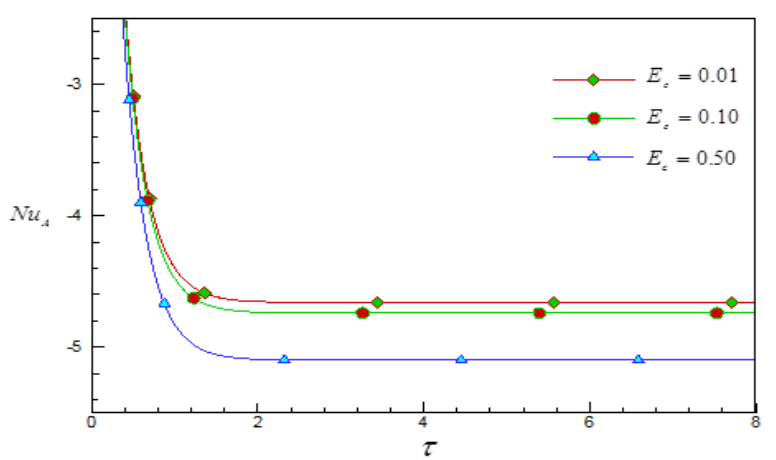

Figure 16(c). Effect of Eckert number $E_{c}$ on average Nusselt number $\mathrm{Nu}_{\mathrm{A}}$

The effects of the Suction velocity $V_{0}$ on the temperature $\theta$, local Nusselt number $N u_{L}$ and on the average Nusselt number $N u_{L}$ are plotted in Figs.17(a)-(c) respectively. It has shown that with the increasing effect of $V_{0}$, the temperature $\theta$, local and average Nusselt number $N u_{L}$ and $N u_{L}$ showed decreasing effects.

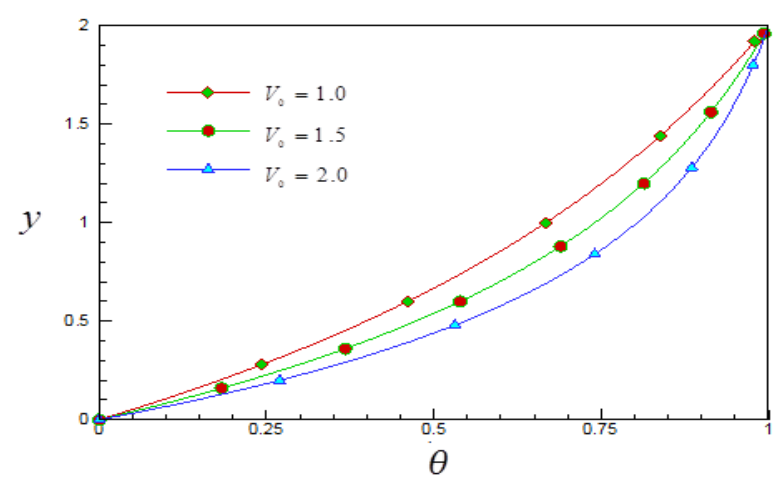

Figure 17(a). Effect of Suction velocity $V_{0}$ on temperature profile $\theta$

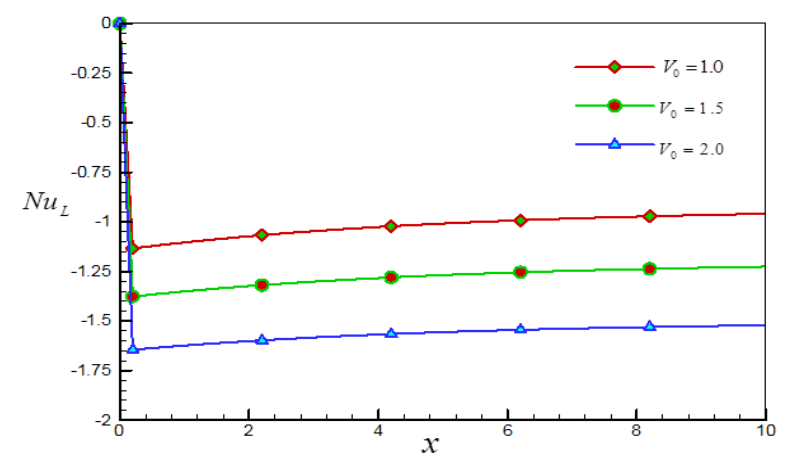

Figure 17(b). Effect of Suction velocity $V_{0}$ on local Nusselt number $\mathrm{Nu} u_{L}$

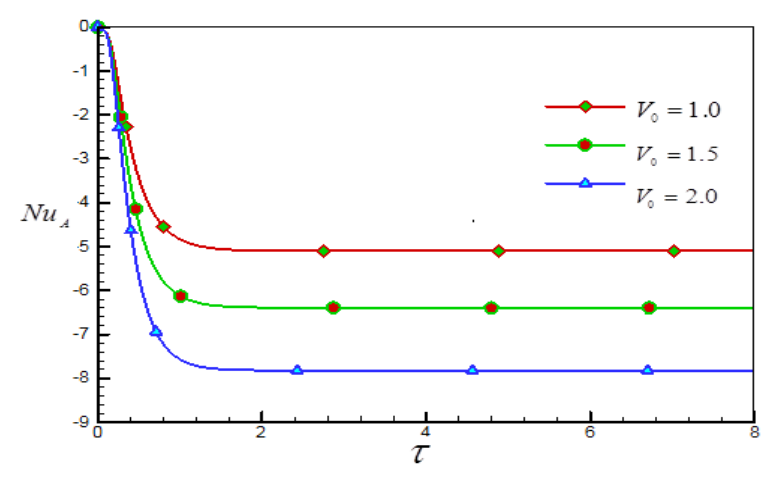

Figure 17(c). Effect of Suction velocity $V_{0}$ on average Nusselt number $\mathrm{Nu}{ }_{\mathrm{A}}$

\section{Conclusions}

The characteristics of two horizontal parallel Riga plates in the presence of Hall and ion slip currents effects have been investigated numerically. The main findings of the study are as follows:

i). Primary velocity profile $u$ increases for the increasing values of pressure gradient parameter $\alpha$,

Hall parameter $\beta_{e}$ and modified Hartmann number $H_{r}$, while it has a decreasing effects with the increase of Ion-slip parameter $\beta_{i}$. The suction velocity $V_{0}$ has a cross effect where the skin friction is increased as the suction velocity $V_{0}$ is increased.

ii). Secondary velocity profile $W$ decreases for the increasing values of Hall parameter $\beta_{e}$, Ion-slip parameter $\beta_{i}$ and modified Hartmann number $H_{r}$. The suction velocity $V_{0}$ shows a cross effect where the skin friction coefficient is increased as the suction velocity $V_{0}$ is decreased.

iii). The temperature profile increases with the rising values of Hall parameter $\beta_{e}$, Ion-slip parameter $\beta_{i}$ and modified Hartmann number $H_{r}$, where as the local and average Nusselt numbers shows the reverse effects.

\section{Nomenclature}

$\tilde{u}$ : Velocity components along $\tilde{x}$-axis

$\widetilde{v}$ : Velocity components along $\tilde{y}$-axis

$\widetilde{w}$ : Velocity components along $\tilde{Z}$ - axis

$\widetilde{t}$ : Dimensional time

$\omega_{e}$ : Cyclotron frequency 
$\tau_{e}:$ Electron Collision time

$\beta_{e}:$ Hall parameter

$\beta_{i}$ : Ion-slip parameter

$\alpha$ : Dimensionless pressure gradient

$\rho$ : Density of the fluid

$\sigma$ : Conductivity of the fluid

$\tau$ : Maximum time

$E_{c}:$ Eckert number

$v$ : Kinematic viscosity of the fluid

$\mu$ : Coefficient of viscosity of the fluid

$u$ : Dimensionless velocity component in $\mathrm{x}$-axis

$V$ : Dimensionless velocity component in y-axis

$B$ : Magnetic field vector

$B_{0}$ : Constant magnetic field vector

$V_{0}$ : Suction Velocity

$\mathrm{k}$ : Thermal conductivity of the fluid

$c_{p}$ : Specific heat capacity at constant pressure

$J_{0}$ : Current density

$\tilde{T}:$ Temperature of the fluid

$\tilde{T}_{w}$ : Constant temperature near the plate

$\tilde{T}_{\infty}:$ Temperature outside of the boundary layer

$\Delta t$ : Time increment

$t$ : Dimensionless time

$\theta$ : Dimensionless temperature

$H_{r}$ : Hartmann number

$P_{r}$ : Prandtl number

$\tau_{*_{L}}:$ Local shear stress

$N u_{L} \quad$ : Local Nusselt number

$\tau_{*_{A}}:$ Average shear stress

$N u_{A}$ : Average Nusselt number

\section{REFERENCES}

[1] A. Gailitis, and O. Leilausis, On a possibility to reduce the hydro dynamical resistance of a plate in an electrode. Appl. Magnetohydrodyn, Vol.12, pp. 143-146, (1961).

[2] Timothy W. Berger, John Kim, Changhoon Lee and Junwoo Lim, Turbulent boundary layer control utilizing the Lorentz force, Physics of Fluids 12, 631 ,(2000); https://doi.org/10.1063/1.870270.

[3] A. Pantokratoras, E. Magyari, MHD free-convection boundary layer flow from a Riga plate. J.Eng. Maths, Vol.64(3), pp. 303-315, (2009).

[4] L.Wahidunnisa, K.Subbarayudu, S.Suneetha, Effect of viscous dissipation over a Riga plate in a nano fluid with heat source/sink. IJTIMES, Vol.4, Issue.6,( 2016).

[5] Z. Iqbal, Ehtsham Azhar, Zaffar Mehmood and E.N. Maraj, Melting heat transport of nanofluidic problem over a Riga

plate with erratic thickness: Use of Keller Box scheme, Results in Physics, Vol.7, pp.3648-3658,( 2017).

[6] Ayub M, Abbas T, Bhatti MM., Inspiration of slip effects on electromagneto hydrodynamics (EMHD) nano fluid flow through a horizontal Riga plate. Eur Phys J Plus 16193, (2016).

[7] Adeel Ahmed, SaleemAsghar and SumairaAfzal, Flow of nanofluid past a Riga plate. Journal of Magnetisnand Magnetic Materials.Vol.420, pp. 44-48, (2016).

[8] Emad M. Aboeldahab and Elsayed M. E. Elbarbary, Hall current effect on magneto-hydrodynamic free convection flow past a semi-infinite vertical plate with mass transfer. Int. J. Engg. Sci.,Vol 39, pp.1641-1652,(2001).

[9] V. Javeri, Combined influence of Hall effect, ion slip, viscous dissipation and Joule heating on MHD heat transfer in a channel.Vol.8, pp.193-202, (1975).

[10] A.H. Eraslan, Temperature distributions in MHD channels with Hall Effect. AIAAJ, Vol.7, pp.186 -188, (1969).

[11] Mohamed A. Seddeek and Emad M. Aboeldahab, Radiative effects on unsteady MHD free convection with Hall current near an infinite vertical porous plate.IJMMS, Vol.26, pp.249-255, (2001).

[12] Lokenath Debnath, Sukomal Chandra Ray and Anddi Kumar Chatterjee, Effects of Hall Current on Unsteady Hydromagnetic Flow past a Porous Plate in a Rotating Fluid System.ZAMM,Vol.59, pp.469-471, (1979).

[13] M. A. Al-Nimr and S. Masoud, Unsteady free convection flow over a vertical flat plate immersed in a porous medium, Fluid Dynamics Research, Vol.23, pp.153-160, (1998).

[14] M. Veera Krishna, N. AmeerAhamad, Ali J. Chamkha ,Hall and ion slip effects on unsteady MHD free convective rotating flow through a saturated porous medium over an exponential accelerated plate.Alexandria Engineering Journal , Vol.59, pp.565-577, (2020).

[15] D. Angirasa and G.P. Peterson, Natural Convection Heat Transform from an Isothermal Vertical Surface to a Fluid Saturated Thermally Stratified Porous Medium. Int. J. Heat Mass Transfer, Vol. 14(8), pp. 4329-4335,(1997).

[16] B.V.R.Kumar, P. Singh, Effect of Thermal Stratification on Free Convection in a Fluid Saturated Porous Enclosure. Numer. Heat Transfer, Vol. 34, pp. 343-356, (1999).

[17] Hazem Ali Attia, Unsteady Couette Flow with Heat Transfer in a Viscoelastic Fluid Considering the Ion Slip. Journal of the Korean Physical Society,Vol. 47, No. 5, pp. 809-817, (2005).

[18] Hazem A. Attia , Unsteady MHD Couette Flow with Heat Transferin the Presence of Uniform Suction and Injection. Mechanics and Mechanical Engineering, Vol. 12, No. 2, pp. 165-176, (2008).

[19] V.Ravi Kumar, M.C.Raju ,G.S.S.Raju,MHD Three Dimensional Couette Flow past aPorous Plate with Heat Transfer. IOSR Journal of Mathematics (IOSRJM), ISSN: 2278-5728 Vol. 1, Issue 3, pp. 03-09, (2012).

[20] Isah BalaYabo, Basant Kumar Jha, Jeng-Eng Lin,On a Couette Flow of Conducting Fluid. International Journal of 
Theoretical and Applied Mathematics, Vol. 4(1), pp.8-21, (2018).

[21] S Harisingh Naik, M V Ramana Murthy, K. Rama Rao ,The effect of Hall current on an unsteady MHD free convective Couette flow between two permeable plates in the presence of thermal radiation. IJCER, ISSN 2250 - 3005, Vol. 04, Issue. 7, (2014).

[22] Victor M. Job and Sreedhara Rao Gunakala, Unsteady MHD Free Convection Couette Flow Through a Vertical Channel in the Presence of Thermal Radiation With Viscous and Joule Dissipation Effects: Using Galerkin's Finite Element Method. IJAIEM, Vol.2, Issue 9, ISSN 2319 -4847, (2013).
[23] Jirapud Limthanakul and Nopparat Pochai, A Two-dimensional Mathematical Model for Long-term Contaminated Groundwater Pollution Measurement around a Land Fill. Mathematics and Statistics, 8(1), pp. 61-74, 2020. DOI: $10.13189 / \mathrm{ms} .2020 .080107$.

[24] Yulia Koroleva, Qualitative Properties of the Solution to Brinkman-Stokes System Modelling a Filtration Process. Mathematics and Statistics, 5(4), 143-150, 2017. DOI: 10.13189/ms.2017.050402.

[25] N. Pochai and N. Pongnu, Numerical solution of groundwater measurement using alternating direction methods. Journal of Interdisciplinary Mathematics, Vol 20, No. 2, 513-541, (2017). 